\title{
Reduction of Proximity Effect in Coil Using Magnetoplated Wire
}

\author{
Tsutomu Mizuno ${ }^{1}$, Shigemi Enoki ${ }^{2}$, Takashi Asahina ${ }^{1}$, Takayuki Suzuki ${ }^{1}$, Masahiro Noda ${ }^{1}$, and \\ Hiroki Shinagawa ${ }^{2}$
}

${ }^{1}$ The Faculty of Engineering, Shinshu University, Nagano 380-8553, Japan

${ }^{2}$ Shinkawa Sensor Technology Inc., Higashi-Hiroshima 739-0153, Japan

\begin{abstract}
The upgrading of the efficiency of transformers and increase in the quality factor of inductors are required. For this purpose, it is necessary to reduce copper loss. Reducing the winding $\mathrm{AC}$ resistance is one method of reducing copper loss. Therefore, we propose the use of magnetoplated wire (MPW) for coil windings. An MPW is a copper wire ( $\mathrm{Cu}$ wire) whose circumference is plated with a magnetic thin film. The resistances due to the proximity effect of $\mathrm{Cu}$ wire and MPW coils at a frequency of $f=1 \mathrm{MHz}$, which have a diameter of $D=4.54 \mathrm{~mm}$, a wire diameter of $d=0.09 \mathrm{~mm}$, and a number of turns of $N=102$, are $4.2 \Omega$ and $2.1 \Omega$, respectively; thus, the proximity effect decreases by half.
\end{abstract}

Index Terms-AC resistance, copper wire, magnetoplated wire, proximity effect, skin effect.

\section{INTRODUCTION}

$\mathbf{T}$ HE upgrading of the efficiency of transformers and increase in the quality factor of inductors are required. For this purpose, it is necessary to reduce copper loss [1]. Reducing the winding $\mathrm{AC}$ resistance is one method of reducing copper loss.

We proposed the use of magnetoplated wire (MPW) for coil winding [2]. An MPW is a copper wire whose circumference is plated with a magnetic thin film. An advantage of using the MPW is that its proximity effect decreases to a lower level than that in the case of copper wire (Cu wire) [3]. Therefore, the AC resistance of an MPW coil is lower than that of a $\mathrm{Cu}$ wire coil.

It is necessary to quantitatively that show the proximity effect is reduced by the use of MPW. Thus, we measured the resistance of $\mathrm{Cu}$ wire and MPW coils, and compared the measured values with calculated values obtained by the finite element method (FEM) [4]. In addition, the reduction in the proximity effect is clarified by comparing the resistance with that of the $\mathrm{Cu}$ wire coil. The following points concerning the reduction of the proximity effect by the use of the MPW are discussed:

1) comparison of measured and calculated values;

2) comparison of resistances due to proximity effect.

\section{StRucture AND MAGNETIC CHARACTERISTICS OF MPW}

\section{A. Structure of $M P W$}

Fig. 1 shows the structures of a $\mathrm{Cu}$ wire and an MPW. The MPW has a copper core plated with magnetic thin films (Fe and Ni) [3]. The thicknesses of the $\mathrm{Fe}$ and $\mathrm{Ni}$ thin films are $1 \mu \mathrm{m}$ and $0.05 \mu \mathrm{m}$, respectively, as shown in Fig. 1(b). The Ni film is plated for ease of soldering.

\section{B. Magnetic Characteristic of MPW}

Fig. 2 shows the $B-H$ loop of MPW. We measured the $B-H$ loop of MPW having $10 \mathrm{~mm}$ length with the vibrating sample magnetometer (Riken Denshi BVH-55). The magnetic field is affected MPW longitudinally. The maximum value of the field

Digital Object Identifier 10.1109/TMAG.2007.893716

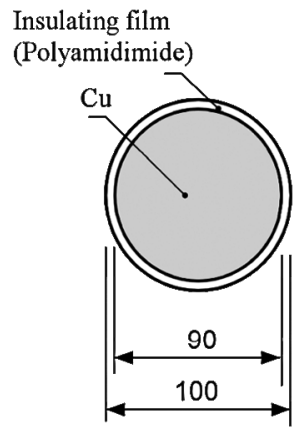

(a)

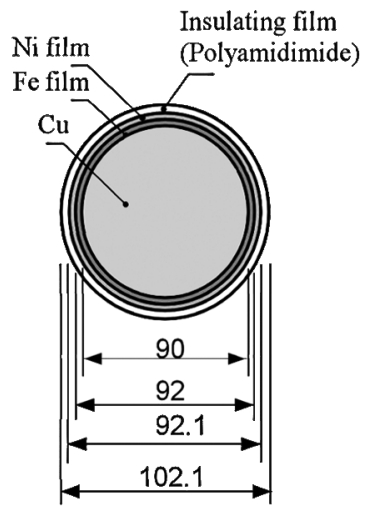

(b)
Fig. 1. Structures of $\mathrm{Cu}$ wire and MPW (unit: $\mu \mathrm{m}$ ). (a) $\mathrm{Cu}$ wire. (b) MPW.

strength obtained by FEM affected MPW is $100 \mathrm{~A} / \mathrm{m}$ under the condition of the measurement of the coil resistance. In this study, MPW was affected the magnetic field of $\pm 80 \mathrm{~A} / \mathrm{m}$ that was the most approximate to $\pm 100 \mathrm{~A} / \mathrm{m}$ from the specification of BVH-55. We assumed the following two matters.

1) Fe thin film is isotropy.

2) The diamagnetic field is disregarded because the demagnatiging factor of MPW that has the diameter of $92 \mu \mathrm{m}$ and the length of $10 \mathrm{~mm}$ is $10^{-3}$ or less [5].

The relative permeability of MPW, $\mu_{\mathrm{r}}$, was 100 shown in Fig. 2.

\section{Method of CALCUlation}

\section{A. Structure of Coils}

Fig. 3 shows the structure of $\mathrm{Cu}$ wire and MPW coils. They are made of the wires shown in Fig. 1. They have an air coil and a coil diameter of $D=4.54 \mathrm{~mm}$ and a number of turns of $N=102$.

\section{B. Condition of FEM Analysis}

Table I lists the conditions of FEM [4], [6]. We analyzed the model of coil shown in Fig. 3 using Maxwell ver. 11. The number of elements of MPW coil was 500000 to analyze the eddy current in the Fe thin film in detail. 


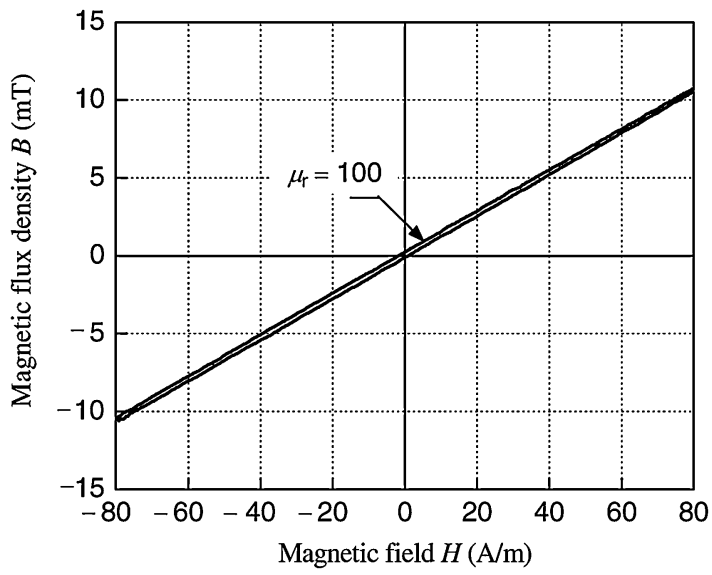

Fig. 2. $B-H$ loop of MPW (measured with Riken Denshi BHV-55).

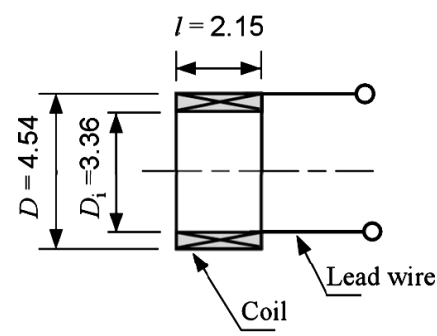

Fig. 3. Structure of coil (unit: $\mathrm{mm}$ ).

TABLE I

CONDITIONS OF FEM

\begin{tabular}{ll}
\hline \hline Item & Content \\
\hline Software & Maxwell ver. 11 (Ansoft co. Ltd) \\
Analysis condition & Cylindrical coordinate \\
& Eddy current solver $(I=1 \mathrm{~mA})$ \\
& $R 70 \times Z 60 \mathrm{~mm}$ \\
Analysis space & $0.5 \%$ or less \\
Error & $50,000(\mathrm{Cu}$ wire $), 500,000(\mathrm{MPW})$ \\
Number of elements & Copper $\left(\varepsilon_{\mathrm{r}}=1, \rho=0.0172 \mu \Omega \cdot \mathrm{m}, \mu_{\mathrm{r}}=0.999991\right)$ \\
Material properties & Fe $\left(\varepsilon_{\mathrm{r}}=1, \rho=0.098 \mu \Omega \cdot \mathrm{m}, \mu_{\mathrm{r}}=100\right)$ \\
& Air $\left(\varepsilon_{\mathrm{r}}=1, \rho=\infty \mu \Omega \cdot \mathrm{m}, \mu_{\mathrm{r}}=1\right)$ \\
\hline \hline
\end{tabular}

\section{COMPARISON OF PROXIMITY EFFECT}

\section{A. Comparison Between Measured and Calculated Resistances}

Fig. 4 shows the comparison between the measured and calculated resistances and inductances of $\mathrm{Cu}$ wire coil, and Fig. 5 shows the comparison between the measured and calculated resistances and inductances of MPW coil. We measured the resistance and inductance vs. frequency characteristics with the impedance analyzer (HP $4192 \mathrm{~A}$ ). The resistance $R$ and the inductance $L$ of the coil were measured with the compensation of $R$ and $L$ of the lead wire shown in Fig. 3. The resonant frequencies of $\mathrm{Cu}$ wire and MPW coils were approximately

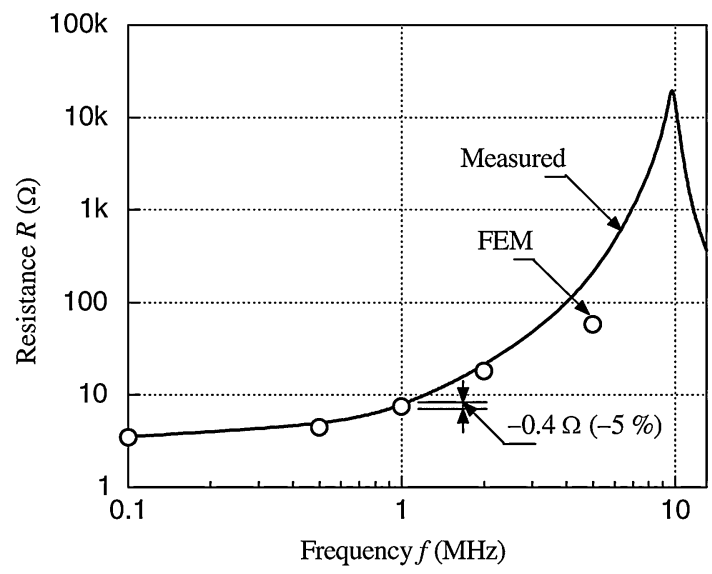

(a)

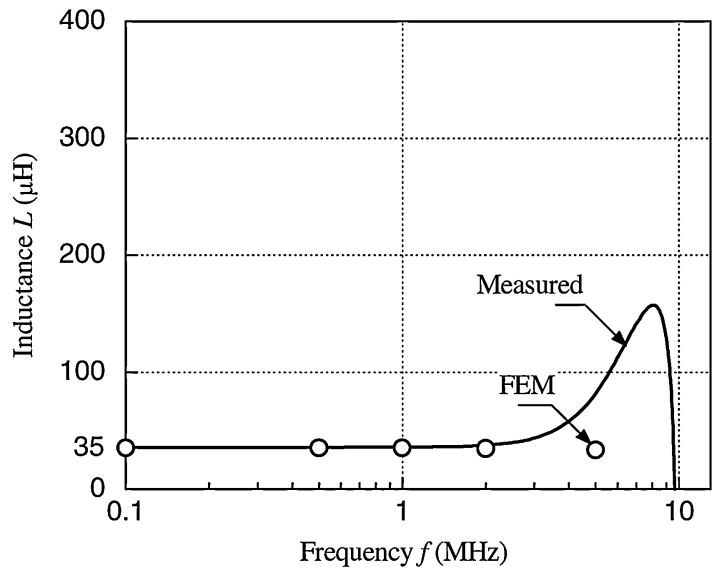

(b)

Fig. 4. Comparison between measured and calculated resistances and inductances of $\mathrm{Cu}$ wire coil. (a) Resistance. (b) Inductance (measured with HP 4192 $\left.\mathrm{A}, \mu_{\mathrm{r}}=0.999991, \rho=0.0172 \mu \Omega \cdot \mathrm{m}\right)$.

$10 \mathrm{MHz}$ shown in Figs. 4(b) and 5(b). In this study, we compared between measured and calculated values at a frequency of $f=1 \mathrm{MHz}$, because it is sufficiently different from the resonant frequency.

The calculation errors of the resistances of the $\mathrm{Cu}$ wire and MPW coils at a frequency of $f=1 \mathrm{MHz}$ were $-5 \%$ and $-12 \%$, respectively; thus, the calculation error of the MPW coil was larger than that of the $\mathrm{Cu}$ wire coil. The calculation accuracy will be higher if the hysteresis loss of the Fe film is considered in the calculation of MPW coil resistance.

\section{B. Comparison of Resistances Due to Proximity Effect}

The coil resistance $R$ is given by

$$
R=R_{\mathrm{dc}}+R_{\mathrm{s}}+R_{\mathrm{p}}
$$

where $R_{\mathrm{dc}}$ is the DC resistance $(\Omega), R_{s}$ is the resistance $(\Omega)$ due to the skin effect, and $R_{\mathrm{p}}$ is the resistance $(\Omega)$ due to the proximity effect.

The resistance of the MPW coil in the frequency range from 0.5 to $2 \mathrm{MHz}$ was decreased by $13 \%-45 \%$ compared with that of the $\mathrm{Cu}$ wire coil, as shown in Figs. 4 and 5. We compare the proximity effect in the MPW coil with that in the $\mathrm{Cu}$ wire coil 


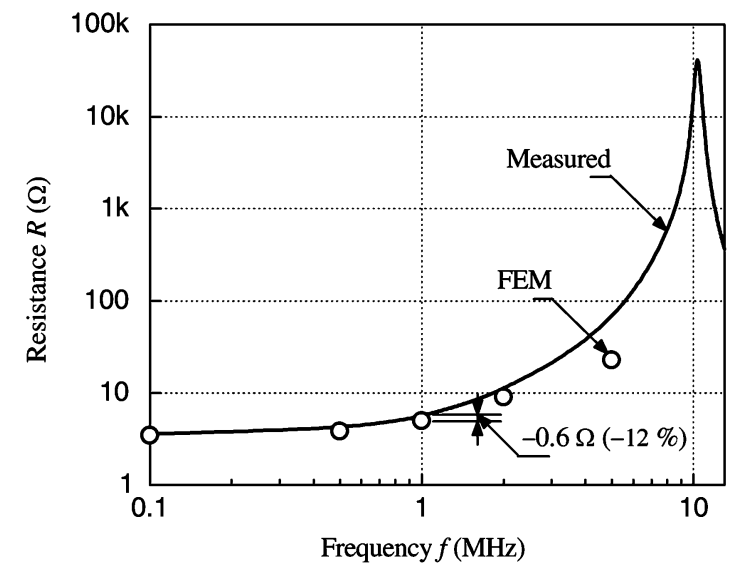

(a)

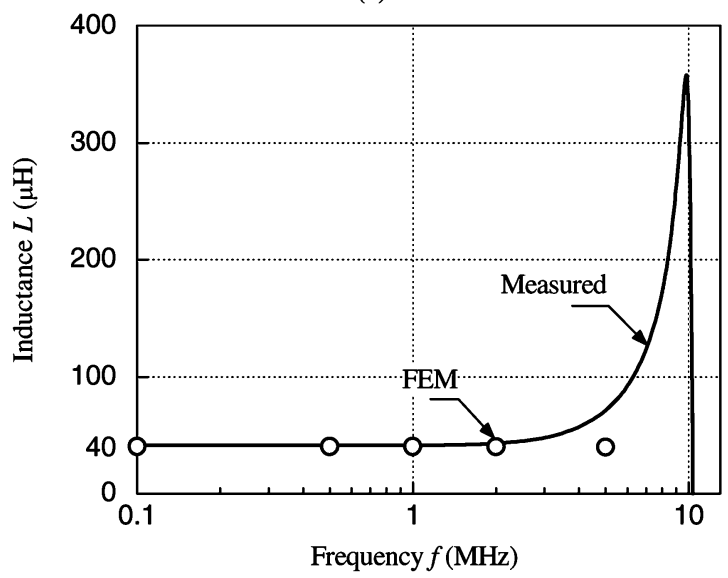

(b)

Fig. 5. Comparison between measured and calculated resistances and inductances of MPW coil. (a) Resistance. (b) Inductance (measured with HP 4192A, $\left.\mu_{\mathrm{r}}=100, \rho=0.098 \mu \Omega \cdot \mathrm{m}\right)$.

in this section. Fig. 6 shows a comparison of coil resistances between the $\mathrm{Cu}$ wire and MPW coils at $f=1 \mathrm{MHz}$. The calculated values of $R_{\mathrm{dc}}+R_{s}$ are shown in this figure. The wire diameter, $d$, was $90 \mu \mathrm{m}$; therefore, the $R_{s}$ at $f=1 \mathrm{MHz}$ was approximately $0 \Omega$. The coil resistances of the $\mathrm{Cu}$ wire and MPW coils at $f=1 \mathrm{MHz}$ were $7.9 \Omega$ and $5.7 \Omega$, respectively; thus, the resistance of the MPW coil decreased by $15 \%$ compared with that of the $\mathrm{Cu}$ wire coil. In addition, the resistances due to the proximity effect, $R_{\mathrm{p}}$, of the $\mathrm{Cu}$ wire and MPW coils were $4.2 \Omega$ and $2.1 \Omega$, respectively; thus, the $R_{\mathrm{p}}$ of the MPW coil was reduced in comparison with that of the $\mathrm{Cu}$ wire coil by half.

\section{CONCLUSION}

The reduction of the proximity effect in an MPW coil was calculated.

\section{A. Comparison Between Measured and Calculated Values}

The calculation errors of $\mathrm{Cu}$ wire and MPW coils were $-5 \%$ and $-12 \%$, respectively; thus, the calculation error of the MPW

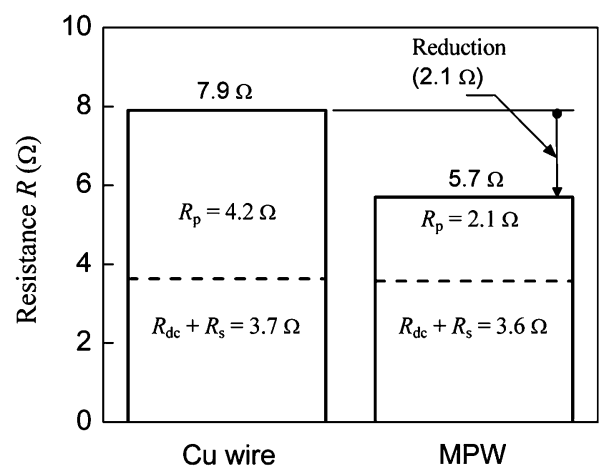

Fig. 6. Comparison of resistances due to proximity effect between $\mathrm{Cu}$ wire and MPW coils $(f=1 \mathrm{MHz})$.

coil was larger than that of the $\mathrm{Cu}$ wire coil, as shown in Figs. 4 and 5.

\section{B. Comparison of Resistances Due to Proximity Effect}

The coil resistances of the COW and MPW coils at $f=1$ $\mathrm{MHz}$ were $7.9 \Omega$ and $5.7 \Omega$, respectively. In addition, the resistances due to the proximity effect, $R_{\mathrm{p}}$, of the $\mathrm{Cu}$ wire and MPW coils were $4.2 \Omega$ and $2.1 \Omega$, respectively; thus, the $R_{\mathrm{p}}$ of the MPW coil was reduced in comparison with that of the COW coil by half, as shown in Fig. 6.

\section{ACKNOWLEDGMENT}

The authors sincerely thank Dr. K. Takeshita of Hitachi HighTech. Electronics Engineering Co., Ltd., and Dr. S. Kishimoto for helpful discussions.

\section{REFERENCES}

[1] J. D. Lavers and V. Bolborici, "Loss comparison in the design of high frequency inductors and transformers," IEEE Trans. Magn., vol. 35, no. 5, pp. 3541-3543, Sep. 1999.

[2] T. Mizuno, T. Hayashi, F. Komeno, T. Asahina, S. Enoki, H. Shinagawa, H. Yamada, S. Watanabe, S. Uehara, T. Kishimoto, and K. Takeshita, "Eddy current displacement sensor using magnet-plated wire," in Technical Meeting on Magnetics, IEE Jpn., 2004, vol. MAG-04-185, pp. 19-24.

[3] S. Yoshimura, S. Yoshihara, T. Shirakashi, E. Sato, and K. Ishii, "Characteristics of high-Q coils composed of Fe-plated Cu wire," in Proc. 1st Magneto Electronics Int. Symp., 1994, pp. 485-487.

[4] Maxwell 2D Version 11, Ansoft Co., Ltd.

[5] Y. Yamamoto and H. Yamada, "Novel analytical equation on magnetic flux distribution and demagnetizing factor of cylindric magnetic core," Trans. IEE Jpn. A, vol. 102, no. 5, pp. 255-262, 1982.

[6] Japan Industrial Standards Committee, "Resistance of copper materials for electrical purposes," JSI C 3001, 3 p., 1981.

Manuscript received October 31, 2006 (e-mail: mizunot@gipwc.shinshu-u. ac.jp). 